\title{
Sigmoid volvulus in pregnancy
}

Kaisha W. 0. ${ }^{1}$ MBChB, M.Med( Surg), Bitta C. ${ }^{2}$ MBChB. E-mail:Wyksa2000@yahoo.com. Affiliation: 1. Department of Human Anatomy, University of Nairobi, 2. Resident, Department of Surgery, University of Nairobi. Correspondence: Dr. W. Kaisha, Department of Human Anatomy, University of Nairobi P.0. Box 368-00202, KNH-Nairobi, Kenya.

\section{Summary}

Sigmoid volvulus in pregnancy is a rare condition that poses a myriad of challenges in diagnosis and management of the patient. It demands on the clinician a high index of suspicion, immediate treatment involving resuscitation and good judgment in handling of the bowel in face of added potential morbidity and mortality to the mother and fetus. We report a case of gangrenous sigmoid volvulus with a viable third trimester pregnancy and discuss potential pitfalls in diagnosis and management.

\section{Introduction}

Sigmoid volvulus complicating pregnancy though reported to be a common cause of intestinal obstruction in pregnancy, is rare (1). For a period of over 100 years since the first reported case in 1885 , only 76 cases had been documented by 1999 (2). None of the reports were found from the African continent. The incidence of intestinal volvulus is known to increase with gestation. The pathology is thought to be due to the lengthening of the mesentery necessitated by the enlarging uterus pushing the pelvic colon into the abdomen (3). Presenting symptoms and signs are similar to those of non-pregnant patients with abdominal pain, vomiting, distension and constipation (4).

The management options are varied and may depend on the stage of pregnancy. When bowel is viable, a conservative approach is recommended for the first trimester, using endoscopic derotation. For recurrent cases, an operation is undertaken in the second trimester when the chances of abortion are the least. In patients who are in the third trimester again a conservative mode of management is advised which may include repeated attempts at derotation for recurrent volvulus. When the pregnancy reaches term elective caesarian and definitive surgery for volvulus can be undertaken simultaneously (1). However in the presence of nonviable colon or unsuccessful reduction of volvulus, sigmoid colectomy and Hartmann's procedure is advised regardless of the gestational age of fetus.

\section{Case report}

A 28 years old, primigravida, 21 weeks gestation by dates, who presented at the emergency department with abdominal pain and obstipation for seven days, abdominal distension for four days and vomiting that was initially bilious and subsequently feculent one day prior to presentation. She had presented five days earlier with the abdominal discomfort and pain and had been treated for urinary tract infection and dyspepsia. She had no medical comorbidities.

Physical examination revealed dehydration, post herpertic skin lesions, fever, mild respiratory distress with a BP of $110 / 70 \mathrm{mmHg}$, pulse rate of $88 / \mathrm{min}$ and respiratory rate of $22 / \mathrm{min}$. The abdomen was grossly distended with obvious visible loops of bowel, and generalized tenderness. The uterus was distinctly palpable with a fundal height of 22 weeks. She had a tympanic note on percussion of upper abdomen and flanks with no audible bowel sounds on auscultation. The fetal heart sounds were not discernible. Digital rectal examination revealed a normal anal and perianal area with normal sphincter tone. The rectum was empty, the pouch of Douglas had no fullness and the uterine adnexae were not appreciated. The other systemic examination was normal. An impression of intestinal obstruction with peritonitis was made.

An ultrasound was able to demonstrate some knotting of the bowel though unable to identify the bowel involved. A viable single fetus of 22 weeks gestation was also observed. Abdominal radiography was not performed at 
earlier review and at second consultation due to fear of fetal exposure to radiation.

Initial fluid rescucitation and nasogastric intubation with initiation of tocolysis was followed by counseling for potential fetal loss and stoma fashioning. At laparotomy a gangrenous sigmoid colon volvulus involved the whole sigmoid colon approximately $50 \mathrm{~cm}$ in length extending $2 \mathrm{~cm}$ from the descending colon to about $3 \mathrm{~cm}$ above the recto-sigmoid junction. (Fig 1). Resection of the gangrenous segment with a Hartmann's procedure was performed. The stoma was fashioned in the left iliac fossa.

The patient had an uneventful post-operative period. A scan performed on the third postoperative day showed that the fetus was viable and corresponding to gestation of 22 weeks. The patient was discharged on the

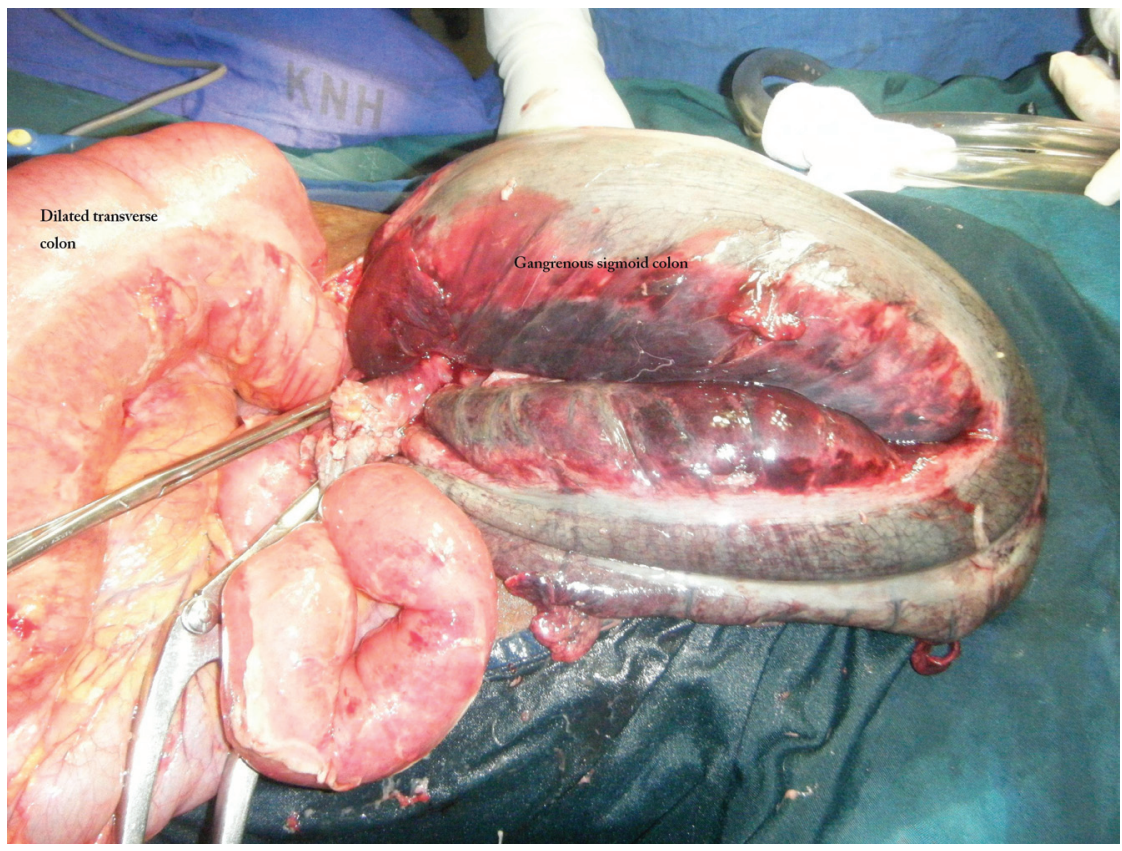

Fig 1: Twisted sigmoid with gangrene and proximal dilated gut fourth post-operative day and counseled on follow up and later closure of stoma. The patient subsequently progressed to term and delivered a healthy baby via emergency caesarian section, having presented in labour with fetal distress. Due to emergency nature of delivery, closure of the stoma was not considered.

\section{Discussion}

Intestinal obstruction in pregnancy is an important pathology that requires prompt attention due to the potential for fetal and maternal morbidity and mortality. Literature differs as to the most common causes in pregnancy with some suggesting sigmoid vovulus and others adhesions $(4,5)$. Most occur in the second and third trimester when increased volume of the uterus and the consequent displacement of abdominal organs cause complications of pathologies which would otherwise escape notice, such as intestinal adhesions, to become manifest (6).

Diagnosis of intestinal obstruction in pregnancy presents various challenges. Despite presenting the classic symptoms of vomiting, distension, abdominal pain and constipation, many times there are delays in diagnosis as these symptoms are explained away as being caused by other pregnancy related conditions (2). Moreover, there is reluctance in obtaining radiographs out of fear of fetal exposure to radiation. In their article on radiation expo-

sure during pregnancy, McCollough et al observed that, the potential biological effects on the fetus were prenatal death, intrauterine growth restriction, small head size, mental retardation, organ malformation, and childhood cancer. The risk was related to the gestational age at the time of exposure, fetal cellular repair mechanisms, and the absorbed radiation dose level. They calculated the radiation levels utilized in radiologic and nuclear medicine and noted that these posed minimal fetal risks and thus concluded that, in cases where examinations provided significant diagnostic information, these should not be withheld from pregnant women (7). However, where ultrasonography is capable of demonstrating adequate clinical information, this should be the preferred mode, since ultrasonography is not associated with radiation exposure (8).

The management of a gravid patient with sigmoid volvulus would largely depend on the stage of pregnancy and viability of the twisted bowel. Various authors report different modalities at different gestational ages. For viable bowel, these include conservative management with colonoscopic detortion and delayed sigmoidectomy with primary anastomosis once the pregnancy has reached maturity. For recurrent cases, an operation in second trimester may be performed when risk of fetal loss are minimum (1). In third trimester, conservative 


\section{Sigmoid volvulus in pregnancy}

Kaisha W., Bitta C. methods are also preferred, since operating in the pelvis at this time, tends to be difficult. However in the presence of nonviable colon or unsuccessful reduction of volvulus, sigmoid colectomy and Hartmann's procedure is advised regardless of the gestational age of fetus (1). The management of a pregnant patient with a stoma is an uncommon event. Few reports on stomas and pregnancy exist in medical literature. It would appear from the available literature that the majority of pregnant patients with stomas should expect no major problems with the stoma and its management during pregnancy (9). However, prolapse seems to be more prevalent in those reported and its management would depend on its severity, pain and discomfort (9). The stoma in itself is not a contraindication to pregnancy. Literature is silent on timing of closure of the stoma (during surgical delivery or later). However, it would appear from assessment of literature on other emergency colonic procedure that closure of the stoma during emergency surgery would be feasible. Specific reference to pregnancy is missing and individualized clinical judgement should be made (10).

\section{Conclusion}

Sigmoid volvulus complicating pregnancy is an uncommon and potentially devastating development that should be suspected with worsening abdominal pain and evidence of bowel obstruction. Prompt intervention is necessary to minimize maternal and fetal morbidity.

\section{References}

1. Alshawi JS. Recurrent sigmoid volvulus in pregnancy: report of a case and review of the literature. Dis Colon Rectum. 2005: 48; 9:1811-3.

2. De U, De KK. Sigmoid volvulus complicating pregnancy. Indian J Med Sci, 2005: 59; 7: 317-318.

3. Ballantyne GH. Review of sigmoid volvulus: clinical patterns and pathogenesis. Dis Colon Rectum. 1982: 25; $494-$ 501

4. Perdue PW, Johnson HW Jr, Stafford PW. Intestinal obstruction complicating pregnancy. Am J Surg. 1992: 164; $4: 384-388$.

5. Connolly MM, Unti JA, Nora PF. Bowel obstruction in pregnancy. Surg Clin North Am 1995: 75; 101-113.

6. Croce P, Rizzitelli E, Montanari G, Galli D, Dedè A .Intestinal volvulus in pregnancy. Clin Ter. 1992: 141; 11:399403.

7. McCollough CH, Schueler BA, Atwell TD, et al. Radiation exposure and pregnancy: when should we be concerned? Radiographics. 2007; 27(4):909-17; discussion 917-8.

8. Siegmann KC, Heuschmid M, Claussen CD. Diagnostic imaging during pregnancy. Dtsch Med Wochenschr. 2009: $134 ; 14: 686-9$

9. Michael C, Thompson J and Cocks P. Stomas and Pregnancy. Aust NZ J Gynae. 1993: 33; 3: 322

10. Madiba T.E and Thomson S.R. The management of sigmoid volvulus. J.R Coll Surg Edinb. 2000: 45; 74-80. 